\title{
Unary patterns under permutations
}

\author{
James Currie \\ Department of Mathematics, University of Winnipeg \\ Florin Manea, Dirk Nowotka, Kamellia Reshadi \\ Kiel University, Department of Computer Science, Kiel, Germany
}

\begin{abstract}
Thue characterized completely the avoidability of unary patterns. Adding function variables gives a general setting capturing avoidance of powers, avoidance of patterns with palindromes, avoidance of powers under coding, and other questions of recent interest. Unary patterns with permutations have been previously analysed only for lengths up to 3 . Consider a pattern $p=\pi_{i_{1}}(x) \ldots \pi_{i_{r}}(x)$, with $r \geq 4, x$ a word variable over an alphabet $\Sigma$ and $\pi_{i_{j}}$ function variables, to be replaced by morphic or antimorphic permutations of $\Sigma$. If $|\Sigma| \geq 3$, we show the existence of an infinite word avoiding all pattern instances having $|x| \geq 2$. If $|\Sigma|=3$ and all $\pi_{i_{j}}$ are powers of a single morphic or antimorphic $\pi$, the length restriction is removed. For the case when $\pi$ is morphic, the length dependency can be removed also for $|\Sigma|=4$, but not for $|\Sigma|=5$, as the pattern $x \pi^{2}(x) \pi^{56}(x) \pi^{33}(x)$ becomes unavoidable. Thus, in general, the restriction on $x$ cannot be removed, even for powers of morphic permutations. Moreover, we show that for every positive integer $n$ there exists $N$ and a pattern $\pi^{i_{1}}(x) \ldots \pi^{i_{n}}(x)$ which is unavoidable over all alphabets $\Sigma$ with at least $N$ letters and $\pi$ morphic or antimorphic permutation.
\end{abstract}

Keywords: Combinatorics on words, avoidable patterns, patterns under

\footnotetext{
A preliminary version of this paper was presented at the International Conference on Developments in Language Theory - DLT 2015 [1].

Email addresses: j.currie@uwinnipeg.ca (James Currie), $\{\mathrm{flm}, \mathrm{dn}, \mathrm{kre}\} @ i n f o r m a t i k . u n i-k i e l . d e(F l o r i n ~ M a n e a$, Dirk Nowotka, Kamellia Reshadi)
} 
permutations

2010 MSC: 68R15

\section{Introduction}

The avoidability of patterns by infinite words is a core topic in combinatorics on words, going back to Thue [2, 3. Important results are surveyed in, e.g., [4, 5].

Recently, a natural generalisation of classical patterns, in which functional 5 dependencies between variables are allowed, has been considered [6, 7, 8. More precisely, patterns consist of word variables, as usual, together with function variables (standing for either morphic or antimorphic extensions of permutations on the alphabet) which act on the words. For example, consider the pattern $x \pi(x) x \pi(x)$ whose instances are words uvuv that consist of four parts of equal length, that is, $|u|=|v|$, where $v$ is the image of $u$ under some permutation of

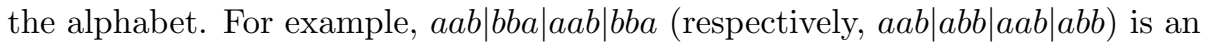
instance of $x \pi(x) x \pi(x)$ for the morphic (respectively, antimorphic) extension of permutation $a \mapsto b$ and $b \mapsto a$.

We note that, while patterns $x^{k}$ describe all repetitions of some exponent $k$, patterns of the type $\pi^{i_{1}}(x) \ldots \pi^{i_{k}}(x)$ describe words that have an intrinsic repetitive structure, hidden by the application of the different iterations of the function $\pi$, which encode of the original root of the repetition.

Patterns with involutions were studied in [6, 7]; motivation for considering involutions includes word reversal and DNA/RNA complementation. The main result obtained was that for each unary pattern with one variable involution, one can identify all alphabets over which it is avoidable. In the more general setting of patterns with permutations, the only results obtained so far (see [8]) regarded cube-like patterns under morphisms or antimorphisms (anti/morphisms, for short) which are powers of a single (variable) permutation, i.e., patterns of the form $\pi^{i}(x) \pi^{j}(x) \pi^{k}(x)$, where $i, j, k \geq 0$. The avoidability of such patterns was completely characterised: for each $\pi^{i}(x) \pi^{j}(x) \pi^{k}(x)$ one can determine exactly the alphabets over which the pattern is avoidable. Contrary to 
both the classical and to the involution settings, where once a pattern is avoidable for some alphabet size it remains avoidable in larger alphabets, a cubic pattern with permutations may become unavoidable over a larger alphabet.

\section{Our contribution}

We extend the results of [8] as follows.

First, we construct a ternary word that avoids all patterns $\pi_{i_{1}}(x) \ldots \pi_{i_{r}}(x)$ where $r \geq 4, x$ a word variable over some alphabet $\Sigma$, with $|x| \geq 2$ and $|\Sigma| \geq 3$,

35 and the $\pi_{i_{j}}$ function variables that may be replaced by anti-/morphic permutations of $\Sigma$. This is the first result where the avoidability of patterns involving more functions, which are not powers of the same initial variable permutation, has been shown; even more, we do not restrict these functions so that all have the same type: we can mix both morphic and antimorphic permutations.

On the down side, the result above only works when we restrict the length of $x$ to be at least 2. However, we also show that such a restriction is needed. Indeed, for each $n \geq 1$ there exists a unary pattern $\pi_{1}(x) \ldots \pi_{n}(x)$ where all functions are powers of the same anti-/morphic permutation $\pi$, i.e., $\pi_{j}=\pi^{i_{j}}$ with $1 \leq j \leq n$, and an integer $N$ such that $\pi^{i_{1}}(x) \ldots \pi^{i_{n}}(x)$ has as instances 45 all the words of length $n$ over an alphabet of size at least $N$; in other words, $\pi^{i_{1}}(x) \ldots \pi^{i_{n}}(x)$ is unavoidable over all alphabets $\Sigma$ with $|\Sigma| \geq N$.

In between these two results, we show that all patterns $\pi^{i_{1}}(x) \ldots \pi^{i_{n}}(x)$ with $n \geq 4$ under anti-/morphic permutations are avoidable in $\Sigma_{3}$. Similarly, all patterns $\pi^{i_{1}}(x) \ldots \pi^{i_{n}}(x)$ under morphic permutations are also avoidable in $\Sigma_{4}$, but

50 not in $\Sigma_{5}$. So, just like in the case of cubes with permutations, there are patterns under anti-/morphic permutations (including the eventually unavoidable patterns we construct) which are avoidable in small alphabets (e.g., in $\Sigma_{3}$ or $\Sigma_{4}$ ) but become unavoidable in larger alphabets. On the other hand, unlike the case of cubes with permutations, where there exist patterns unavoidable in $\Sigma_{2}$ and $\Sigma_{3}$ (e.g., $x \pi(x) \pi^{2}(x)$, see [8]), all unary patterns of length at least 4 under anti-/morphic permutations are avoidable in both $\Sigma_{2}$ (see [7]) and $\Sigma_{3}$, and, in the case of morphic permutations, in $\Sigma_{4}$ as well. Note that 4 is the 
largest integer $i$ such that all patterns of length 4 under morphic permutations are avoidable in $\Sigma_{i}$. \\ 2. Definitions}

We freely use the usual notations of combinatorics on words (see, for instance, 4]). Define alphabets $\Sigma_{k}=\{0, \ldots, k-1\}$ and $\Sigma_{k}^{\prime}=\{1,2, \ldots, k\}$. We use $w^{R}$, to denote the reversal of word $w$.

A morphism $f$ (respectively, antimorphism) of $\Sigma_{k}^{*}$ is defined by its values on letters; $f(u v)=f(u) f(v)$ (respectively, $f(u v)=f(v) f(u)$ ) for all words $u, v \in \Sigma_{k}^{*}$. When we define an anti-/morphism it is enough to define $f(a)$, for all $a \in \Sigma_{k}$. If the restriction of $f$ to $\Sigma_{k}$, is a permutation of $\Sigma_{k}$, we call $f$ an anti-/morphic permutation. Denote by ord $(f)$ the order of $f$, i.e., the minimum positive integer $m$ such that $f^{m}$ is the identity. If $\operatorname{ord}(f)=2$, we call $f$ an involution. If $a \in \Sigma_{k}$ is a letter, the order of $a$ with respect to $f$, denoted $\operatorname{ord}_{f}(a)$, is the minimum number $m$ such that $f^{m}(a)=a$.

A pattern which involves functional dependencies is a term over (word) variables and function variables (where concatenation is an implicit functional constant); a pattern with only one word variable is called unary. For example,

${ }_{75} x \pi(x) \pi(\pi(x)) x=x \pi(x) \pi^{2}(x) x$ is a unary pattern involving the variable $x$ and the function variable $\pi$. An instance of a pattern $p$ in $\Sigma_{k}$ is the result of substituting every variable by a word in $\Sigma_{k}^{+}$and every function variable by a function over $\Sigma_{k}^{*}$. A pattern is avoidable in $\Sigma_{k}$ if there is an infinite word over $\Sigma_{k}$ that does not contain any instance of the pattern.

In this paper, we consider patterns with morphic and antimorphic permutations, that is, all function variables are substituted by morphic or antimorphic permutations only.

The infinite Thue-Morse word $\mathbf{t}$ (see [2]) is defined as $\mathbf{t}=\lim _{n \rightarrow \infty} \phi_{t}^{n}(0)$, for the morphism $\phi_{t}: \Sigma_{2}^{*} \rightarrow \Sigma_{2}^{*}$ where $\phi_{t}(0)=01$ and $\phi_{t}(1)=10$. It is wellknown (see, for instance, 4] ) that $\mathbf{t}$ avoids the patterns $x x x$ (cubes) and $x y x y x$ (overlaps). 
Let $\mathbf{h}$ be the infinite word defined as $\mathbf{h}=\lim _{n \rightarrow \infty} \phi_{h}^{n}(0)$, where $\phi_{h}: \Sigma_{3}^{*} \rightarrow \Sigma_{3}^{*}$ is a morphism due to Thue 2, which was rediscovered and studied also by Hall [9], defined by $\phi_{h}(0)=012, \phi_{h}(1)=02$ and $\phi_{h}(2)=1$. For the simplicity of the

so exposure, if $\mathbf{h}=\prod_{i=0}^{\infty} h_{i}$ with $h_{i} \in \Sigma_{3}$, we define the infinite word $\mathbf{v}$ over $\Sigma_{3}^{\prime}$ as $\mathbf{v}=\prod_{i=0}^{\infty} v_{i}$, with $v_{i}=h_{i}+1$. The infinite word $\mathbf{v}$ (respectively, the word $\mathbf{h}$ ) avoids squares $x x$ and does not contain the factors 121 and 323 (respectively, the factors 010 and 212).

We investigate the factors of an infinite word $\mathbf{g}$ that have the form

$$
\pi_{i_{1}}(x) \pi_{i_{2}}(x) \ldots \pi_{i_{r}}(x)
$$

with $x$ a non-empty word and each $\pi_{i_{j}}$ a morphic or antimorphic permutation

95 for $1 \leq j \leq r$. Replacing $x$ by $\pi_{i_{1}}^{-1}(x)$ and $\pi_{i_{j}}(x)$ by $\pi_{i_{j}}\left(\pi_{i_{1}}^{-1}(x)\right)$ for $1 \leq j \leq r$, this is equivalent to investigating factors of $\mathbf{g}$ of the form $x \pi_{j_{1}}(x) \ldots \pi_{j_{r-1}}(x)$ with $x$ a non-empty word, and each $\pi_{j_{\ell}}$ a morphic or antimorphic permutation for all $1 \leq \ell \leq r-1$.

\section{A general result}

We use the word $\mathbf{v}$ defined above to define the word $\mathbf{u} \in \Sigma_{3}^{\omega}$ given by

$$
\mathbf{u}=\prod_{i=0}^{\infty}\left(0^{v_{3 i}} 1^{v_{3 i+1}} 2^{v_{3 i+2}}\right)
$$

Theorem 1. The word $\mathbf{u}$ has no factor of the form $x \pi_{i}(x) \pi_{j}(x) \pi_{k}(x)$ with $|x| \geq$ 2 and $\pi_{i}, \pi_{j}$ and $\pi_{k}$ are each a morphic or antimorphic permutation.

Proof. (Morphic case) Suppose, to the contrary, that $\mathbf{u}$ has a factor $w=$ $x \pi_{i}(x) \pi_{j}(x) \pi_{k}(x)$ with $|x| \geq 2$, where each $\pi_{r}$ is a morphic permutation. We consider the block structure of $x$; that is, we parse $x$ as

$$
x=a_{1}^{k_{1}} a_{2}^{k_{2}} \cdots a_{n-1}^{k_{n-1}} a_{n}^{k_{n}}
$$

where the $a_{i} \in \Sigma_{3}$, with $a_{\ell} \neq a_{\ell+1}, k_{\ell} \geq 1,1 \leq \ell \leq n$. Certainly, $\pi_{r}(x)$ has the same block structure for each $r$ :

$$
\pi_{r}(x)=\left(\pi_{r}\left(a_{1}\right)\right)^{k_{1}}\left(\pi_{r}\left(a_{2}\right)\right)^{k_{2}} \cdots\left(\pi_{r}\left(a_{n-1}\right)\right)^{k_{n-1}}\left(\pi_{r}\left(a_{n}\right)\right)^{k_{n}}
$$


and letters $\pi_{r}\left(a_{\ell}\right)$ and $\pi_{r}\left(a_{\ell+1}\right)$ are distinct, since $\pi_{r}$ is a permutation.

We consider several cases based on $n, k_{1}$ and $k_{n}$ as follows:

Case 1: $n=1$. This means that $w=a_{1}^{k_{1}}\left(\pi_{i}\left(a_{1}\right)\right)^{k_{1}}\left(\pi_{j}\left(a_{1}\right)\right)^{k_{1}}\left(\pi_{k}\left(a_{1}\right)\right)^{k_{1}}$.

Since $|x| \geq 2$, we have $k_{1} \geq 2$. If $a_{1}=\pi_{i}\left(a_{1}\right)$, then $w$ contains the factor $a_{1}^{k_{1}}\left(\pi_{i}\left(a_{1}\right)\right)^{k_{1}}=a_{1}^{2 k_{1}}$. Since $2 k_{1} \geq 4$, this is impossible; the block lengths in $\mathbf{u}$ are 1,2 or 3 . We conclude that $a_{1} \neq \pi_{i}\left(a_{1}\right)$. Similarly, $\pi_{i}\left(a_{1}\right) \neq \pi_{j}\left(a_{1}\right)$, and $\pi_{j}\left(a_{1}\right) \neq \pi_{k}\left(a_{1}\right)$. It follows that, in the context of $w,\left(\pi_{i}\left(a_{1}\right)\right)^{k_{1}}$ and $\left(\pi_{j}\left(a_{1}\right)\right)^{k_{1}}$ are successive blocks of $\mathbf{u}$; however, this implies that $k_{1} k_{1}$ is a factor of $\mathbf{v}$. Since $\mathbf{v}$ is square-free, this is impossible.

Case 2a: $n>1$, and $k_{1}=3$ or $k_{n}=3$

Suppose $k_{1}=3$. This implies that $a_{n} \neq \pi_{i}\left(a_{1}\right)$; otherwise $w$ contains a block $a_{n}^{k_{n}}\left(\pi_{i}\left(a_{1}\right)\right)^{3}=a_{n}^{k_{n}+3}$, of length 4 or greater. Similarly, $\pi_{i}\left(a_{n}\right) \neq \pi_{j}\left(a_{1}\right)$ and $\pi_{j}\left(a_{n}\right) \neq \pi_{k}\left(a_{1}\right)$. Each $\left(\pi_{i}\left(a_{\ell}\right)\right)^{k_{\ell}}$ and $\left(\pi_{j}\left(a_{\ell}\right)\right)^{k_{\ell}}$ is thus a complete block of $\mathbf{u}$, and $\mathbf{v}$ contains the factor $\left(k_{1} k_{2} \cdots k_{n}\right)^{2}$. This is impossible. Similarly, one argues that $k_{n}=3$ gives a contradiction.

Case 2b: $n>1$, and $k_{1}, k_{n} \leq 2$

If $a_{n}=\pi_{i}\left(a_{1}\right)$ and $\pi_{i}\left(a_{n}\right)=\pi_{j}\left(a_{1}\right)$, then $\mathbf{u}$ contains the factor $a_{n-1} a_{n}^{k_{n}+k_{1}}\left(\pi_{i}\left(a_{2}\right)\right)^{k_{2}} \ldots\left(\pi_{i}\left(a_{n-1}\right)\right)^{k_{n-1}} \pi_{i}\left(a_{n}\right)^{k_{n}+k_{1}}\left(\pi_{j}\left(a_{2}\right)\right)^{k_{2}} \ldots\left(\pi_{j}\left(a_{n-1}\right)\right)^{k_{n-1}} \pi_{j}\left(a_{n}\right)$, and $\mathbf{v}$ contains the square factor $\left(\left(k_{n}+k_{1}\right) k_{2} k_{3} \cdots k_{n-1}\right)^{2}$, which is impossible. Similarly, if $a_{n} \neq \pi_{i}\left(a_{1}\right)$ and $\pi_{i}\left(a_{n}\right) \neq \pi_{j}\left(a_{1}\right)$, then $\mathbf{v}$ contains the factor

$$
a_{1} a_{2}^{k_{2}} \ldots a_{n}^{k_{n}}\left(\pi_{i}\left(a_{1}\right)\right)^{k_{1}}\left(\pi_{i}\left(a_{2}\right)\right)^{k_{2}} \ldots\left(\pi_{i}\left(a_{n}\right)\right)^{k_{n}}\left(\pi_{j}\left(a_{1}\right)\right)^{k_{1}} \pi_{j}\left(a_{2}\right),
$$

and then $v$ contains the factor $\left(k_{2} k_{3} \cdots k_{1}\right)^{2}$, which is again impossible. In conclusion, exactly one of the equations $a_{n}=\pi_{i}\left(a_{1}\right)$ and $\pi_{i}\left(a_{n}\right)=\pi_{j}\left(a_{1}\right)$ holds. Similarly, exactly one of $\pi_{i}\left(a_{n}\right)=\pi_{j}\left(a_{1}\right)$ and $\pi_{j}\left(a_{n}\right)=\pi_{k}\left(a_{1}\right)$ holds.

Case 2bi: $k_{1}, k_{n} \leq 2$, and $n \geq 3$. Suppose that $a_{n}=\pi_{i}\left(a_{1}\right)$ and $\pi_{i}\left(a_{n}\right) \neq$ $\pi_{j}\left(a_{1}\right)$. (The other case is similar.)

Since $\pi_{i}\left(a_{n}\right) \neq \pi_{j}\left(a_{1}\right)$, but

$$
\pi_{i}\left(a_{n-1}\right)\left(\pi_{i}\left(a_{n}\right)\right)^{k_{n}}\left(\pi_{j}\left(a_{1}\right)\right)^{k_{1}} \pi_{j}\left(a_{2}\right)
$$


is a factor of $\mathbf{u}$, we see that $k_{n} k_{1}$ is a factor of $\mathbf{v}$, whence $k_{n} \neq k_{1}$. Since we have already reasoned that $k_{1}, k_{n} \leq 2$, we see that $k_{1}+k_{n}=3$. Now $a_{n-2}\left(a_{n-1}\right)^{k_{n-1}} a_{n}^{3}$ is a factor of $\mathbf{u}$, so that $k_{n-1} \neq 3$. On the other hand, since

$$
\pi_{i}\left(a_{n-2}\right)\left(\pi_{i}\left(a_{n-1}\right)\right)^{k_{n-1}}\left(\pi_{i}\left(a_{n}\right)\right)^{k_{n}} \pi_{j}\left(a_{1}\right)
$$

is a factor of $\mathbf{u}$, and $\pi_{i}\left(a_{n}\right) \neq \pi_{j}\left(a_{1}\right)$, we conclude that $k_{n-1} k_{n}$ is a factor of $\mathbf{v}$; therefore, $k_{n-1} \neq k_{n}$, and since $k_{n}, k_{n-1}<3$, we have $k_{n-1}=3-k_{n}=k_{1}$.

Similar reasoning shows that $k_{2}=k_{n}$. But then

$$
\pi_{i}\left(a_{n-2}\right)\left(\pi_{i}\left(a_{n-1}\right)^{k_{n-1}}\left(\pi_{i}\left(a_{n}\right)\right)^{k_{n}}\left(\pi_{j}\left(a_{1}\right)\right)^{k_{1}}\left(\pi_{j}\left(a_{2}\right)\right)^{k_{2}} \pi_{j}\left(a_{3}\right)\right.
$$

125 is a factor of $\mathbf{u}$, so that $k_{n-1} k_{n} k_{1} k_{2}=\left(k_{1} k_{n}\right)^{2}$ is a factor of $\mathbf{v}$. This is impossible.

Case 2bii: $k_{1}, k_{n} \leq 2$, and $n=2$. We make four subcases, depending on whether $\left(k_{1}, k_{2}\right)=(1,2)$ or $(2,1)$, and on whether $a_{2}=\pi_{i}\left(a_{1}\right), \pi_{i}\left(a_{2}\right) \neq \pi_{j}\left(a_{1}\right)$ and $\pi_{j}\left(a_{2}\right)=\pi_{k}\left(a_{1}\right)$, or alternatively, $a_{2} \neq \pi_{i}\left(a_{1}\right), \pi_{i}\left(a_{2}\right)=\pi_{j}\left(a_{1}\right)$ and $\pi_{j}\left(a_{2}\right) \neq$ $\pi_{k}\left(a_{1}\right)$.

1. $\left(k_{1}, k_{2}\right)=(1,2), a_{2}=\pi_{i}\left(a_{1}\right), \pi_{i}\left(a_{2}\right) \neq \pi_{j}\left(a_{1}\right), \pi_{j}\left(a_{2}\right)=\pi_{k}\left(a_{1}\right)$ :

In this case, $\mathbf{u}$ contains the word

$$
\begin{gathered}
a_{1} a_{2}^{2} \pi_{i}\left(a_{1}\right)\left(\pi_{i}\left(a_{2}\right)\right)^{2} \pi_{j}\left(a_{1}\right)\left(\pi_{j}\left(a_{2}\right)\right)^{2} \pi_{k}\left(a_{1}\right)\left(\pi_{k}\left(a_{2}\right)\right)^{2} \\
=a_{1} a_{2}^{3}\left(\pi_{i}\left(a_{2}\right)\right)^{2} \pi_{j}\left(a_{1}\right)\left(\pi_{j}\left(a_{2}\right)\right)^{3}\left(\pi_{k}\left(a_{2}\right)\right)^{2}
\end{gathered}
$$

so that $\mathbf{v}$ contains a word $\alpha 3213 \beta, \alpha, \beta \in\{1,2,3\}, \beta \geq 2$. In fact, if $\beta=3$, then $\mathbf{v}$ contains the square $3^{2}$. Assume then that $\beta=2$. Thus 32132 is a factor of $\mathbf{v}$; however, 32132 has no right extension in $\mathbf{v}$, since 321321 and 321322 end in squares, while 321323 ends in 323. This is impossible.

2. $\left(k_{1}, k_{2}\right)=(2,1), a_{2}=\pi_{i}\left(a_{1}\right), \pi_{i}\left(a_{2}\right) \neq \pi_{j}\left(a_{1}\right), \pi_{j}\left(a_{2}\right)=\pi_{k}\left(a_{1}\right)$ :

In this case, $\mathbf{u}$ contains the word

$$
\begin{gathered}
a_{1}^{2} a_{2}\left(\pi_{i}\left(a_{1}\right)\right)^{2} \pi_{i}\left(a_{2}\right)\left(\pi_{j}\left(a_{1}\right)\right)^{2} \pi_{j}\left(a_{2}\right)\left(\pi_{k}\left(a_{1}\right)\right)^{2} \pi_{k}\left(a_{2}\right) \\
=a_{1}^{2} a_{2}^{3} \pi_{i}\left(a_{2}\right)\left(\pi_{j}\left(a_{1}\right)\right)^{2}\left(\pi_{j}\left(a_{2}\right)\right)^{3} \pi_{k}\left(a_{2}\right)
\end{gathered}
$$


so that $\mathbf{v}$ contains a word $\alpha 3123 \beta, \alpha, \beta \in\{1,2,3\}, \alpha \geq 2$. In fact, if $\alpha=3$, then $\mathbf{v}$ contains $3^{2}$. Assume then that $\alpha=2$, so that $\mathbf{v}$ contains 23123. Since $\mathbf{v}$ is recurrent, 23123 must have a left extension in $\mathbf{v}$; however, none of 123123,223123 and 323123 is a possible factor of $\mathbf{v}$.

3. $\left(k_{1}, k_{2}\right)=(1,2), a_{2} \neq \pi_{i}\left(a_{1}\right), \pi_{i}\left(a_{2}\right)=\pi_{j}\left(a_{1}\right), \pi_{j}\left(a_{2}\right) \neq \pi_{k}\left(a_{1}\right)$ :

In this case, $w$ contains the word

$$
\begin{gathered}
a_{1} a_{2}^{2} \pi_{i}\left(a_{1}\right)\left(\pi_{i}\left(a_{2}\right)\right)^{2} \pi_{j}\left(a_{1}\right)\left(\pi_{j}\left(a_{2}\right)\right)^{2} \pi_{k}\left(a_{1}\right)\left(\pi_{k}\left(a_{2}\right)\right)^{2} \\
\quad=a_{1} a_{2}^{2} \pi_{i}\left(a_{1}\right)\left(\pi_{i}\left(a_{2}\right)\right)^{3}\left(\pi_{j}\left(a_{2}\right)\right)^{2} \pi_{k}\left(a_{1}\right)\left(\pi_{k}\left(a_{2}\right)\right)^{2}
\end{gathered}
$$

so that $v$ contains a word $\alpha 21321 \beta$. No left extension of this word is a factor of $\mathbf{v}$.

4. $\left(k_{1}, k_{2}\right)=(2,1), a_{2} \neq \pi_{i}\left(a_{1}\right), \pi_{i}\left(a_{2}\right)=\pi_{j}\left(a_{1}\right), \pi_{j}\left(a_{2}\right) \neq \pi_{k}\left(a_{1}\right)$ : In this case, $w$ contains the word

$$
\begin{gathered}
a_{1}^{2} a_{2}\left(\pi_{i}\left(a_{1}\right)\right)^{2} \pi_{i}\left(a_{2}\right)\left(\pi_{j}\left(a_{1}\right)\right)^{2} \pi_{j}\left(a_{2}\right)\left(\pi_{k}\left(a_{1}\right)\right)^{2} \pi_{k}\left(a_{2}\right) \\
\quad=a_{1}^{2} a_{2}\left(\pi_{i}\left(a_{1}\right)\right)^{2}\left(\pi_{i}\left(a_{2}\right)\right)^{3} \pi_{j}\left(a_{2}\right)\left(\pi_{k}\left(a_{1}\right)\right)^{2} \pi_{k}\left(a_{2}\right)
\end{gathered}
$$

so that $v$ contains a word $\alpha 12312 \beta$. No right extension of this word is a factor of $\mathbf{v}$.

We see that $w$ contains no instance $x \pi_{i}(x) \pi_{j}(x) \pi_{k}(x)$ with $|x| \geq 2$ where each $\pi_{r}$ is a morphic permutation.

(Antimorphic case) Suppose, for the sake of getting a contradiction, that $\mathbf{u}$ has a factor $w=x \pi_{i}(x) \pi_{j}(x) \pi_{k}(x)$ with $|x| \geq 2$, where one of the $\pi_{r}$ is an antimorphic permutation.

For notational simplicity, we will suppose that $\pi_{i}$ is antimorphic; the other cases are similar.

We consider the block structure of $x$ :

$$
x=a_{1}^{k_{1}} a_{2}^{k_{2}} \cdots a_{n-1}^{k_{n-1}} a_{n}^{k_{n}}
$$

where the $a_{i} \in \Sigma_{3}$, with $a_{\ell} \neq a_{\ell+1}, k_{\ell} \geq 1,1 \leq \ell \leq n$. Since $\pi_{i}$ is antimorphic,

$$
\pi_{i}(x)=\left(\pi_{i}\left(a_{n}\right)\right)^{k_{n}}\left(\pi_{i}\left(a_{n-1}\right)\right)^{k_{n-1}} \cdots\left(\pi_{i}\left(a_{2}\right)\right)^{k_{2}}\left(\pi_{i}\left(a_{1}\right)\right)^{k_{1}} .
$$


If $k_{n}=3$, then $\mathbf{u}$ has the factor $a_{n}^{k_{n}} \pi_{i}\left(a_{n}\right)^{k_{n}}$, and either $\mathbf{u}$ has a block of length 6 (if $a_{n}=\pi_{i}\left(a_{n}\right)$ ), or $\mathbf{v}$ has a factor 33 ; both cases are impossible.

If $k_{n}=2$, we make cases based on $n$ : If $n=1$, then $w=a_{1}^{2} \pi_{i}\left(a_{1}^{2}\right) \pi_{j}\left(a_{1}^{2}\right) \pi_{k}\left(a_{1}^{2}\right)$, and the factor $\pi_{i}\left(a_{1}^{2}\right) \pi_{j}\left(a_{1}^{2}\right)$ of $w$ implies that either $\mathbf{u}$ has a block of length 4 , or $\mathbf{v}$ has a factor 22 ; both cases are impossible.

If $n>1$, then factor $a_{n-1}^{k_{n}-1} a_{n}^{k_{n}} \pi_{i}\left(a_{n}^{k_{n}}\right) \pi_{i}\left(a_{n-1}^{k_{n-1}}\right)$ gives the same contradiction.

We conclude that $k_{n}=1$. Since $|x| \geq 2$, we have $n \geq 2$. If $a_{n} \neq \pi_{i}\left(a_{n}\right)$, then the factor $a_{n-1}^{k_{n-1}} a_{n}^{1} \pi_{i}\left(a_{n}^{1}\right) \pi_{i}\left(a_{n-1}^{k_{n-1}}\right)$ of $w$ implies that 11 is a factor of $\mathbf{v}$, which is impossible. We conclude that $a_{n}=\pi_{i}\left(a_{n}\right)$.

Suppose $|x| \geq 3$. If $k_{n-1}=1$, then $w$ contains $a_{n-2}^{k_{n-2}} a_{n-1}^{1} a_{n}^{2} \pi_{i}\left(a_{n-1}^{1}\right) \pi_{i}\left(a_{n-2}\right)$, so that $\mathbf{v}$ has the factor 121 . This is impossible. Thus $k_{n-1}>1$. This forces $\mathbf{u}$ to contain a block $a_{n-1}^{y} a_{n}^{2} \pi_{i}\left(a_{n-1}^{z}\right)$, where $y, z \geq 2$ and $y 2 z$ is a factor of $\mathbf{v}$. However, then $\mathbf{v}$ has 22 or 323 as a factor, which is impossible. We conclude that $|x|=2$. It follows that $n=2, k_{1}=k_{2}=1$.

Write $w=a_{1} a_{2} b_{1} b_{2} c_{1} c_{2} d_{1} d_{2}$, each $a_{i}, b_{i}, c_{i}, d_{i} \in \Sigma_{3}$, and $a_{1} \neq a_{2}, b_{1} \neq b_{2}$, $c_{1} \neq c_{2}, d_{1} \neq d_{2}$. We have arrived at this case by considering the word $x \pi_{i}(x)$, assuming that $\pi_{i}$ is antimorphic. If, instead, $\pi_{i}$ is morphic and $\pi_{j}$ is antimorphic, (resp., $\pi_{i}$ and $\pi_{j}$ are morphic, $\pi_{k}$ is antimorphic) the same analysis goes through considering the word $\pi_{i}(x) \pi_{j}(x)$ (resp., $\pi_{j}(x) \pi_{k}(x)$ ).

We must have $a_{2}=b_{1}$, or $\mathbf{v}$ contains the square 11. Similarly, $b_{2}=c_{1}$. Now, however, $\mathbf{v}$ contains the square 22 . This is a contradiction.

Consequently, $\mathbf{u}$ has no factor of the form $\pi_{\ell}(x) \pi_{i}(x) \pi_{j}(x) \pi_{k}(x)$ with $|x| \geq$ 2 and $\pi_{i}, \pi_{j}$ and $\pi_{k}$ are each a morphic or antimorphic permutation. This means that $\mathbf{u}$ has not factor that contains, at its turn, an instance of a pattern $\pi_{\ell}(x) \pi_{i}(x) \pi_{j}(x) \pi_{k}(x)$ with $|x| \geq 2$ and $\pi_{i}, \pi_{j}$ and $\pi_{k}$ are each a morphic or antimorphic permutation. So, the following general theorem follows.

Theorem 2. The word $\mathbf{u}$ has no factor of the form $\pi_{i_{1}}(x) \pi_{i_{2}}(x) \ldots \pi_{i_{r}}(x)$ with $|x| \geq 2, r \geq 4$, and $\pi_{i_{j}}$ is a morphic or antimorphic permutation for $1 \leq j \leq r$. 


\section{Avoidability for small alphabets}

\subsection{Ternary alphabets}

We now show that all patterns of length at least 4 under anti-/morphisms which are powers of the same permutation are avoidable in $\Sigma_{3}$. More precisely, we show that for each pattern $\mathcal{P}=x \pi^{i}(x) \pi^{j}(x) \pi^{k}(x)$ there exists an infinite word (that depends on $\mathcal{P}$ ) that does not contain any instance of $\mathcal{P}$ with $\pi$ an anti-/morphic permutation of $\Sigma_{3}$.

Let us note from the beginning that the permutations of $\Sigma_{3}$ are either cycles (i.e., $\operatorname{ord}(a)=3$ for all $a \in \Sigma_{3}$ ) or involutions (i.e., $\operatorname{ord}(a) \leq 2$ for all $\left.a \in \Sigma_{3}\right)$.

We use as a basic lemma the following corollary of Theorem 1 .

Corollary 1. The word $\mathbf{u}$ has no factor of the form $x \pi^{i}(x) \pi^{j}(x) x$, where $\pi$ is an anti-/morphic permutation of $\Sigma_{3}$.

Proof. By Theorem 1 we know that $\mathbf{u}$ has no factor of the form $x \pi^{i}(x) \pi^{j}(x) x$ with $|x| \geq 2$, by just taking in the statement of the theorem $\pi_{i}=\pi^{i}, \pi_{j}=\pi^{j}$, and $\pi_{k}$ the identity on $\Sigma_{3}$. We assume, for the sake of a contradiction, that $\mathbf{u}$ has a factor $x \pi^{i}(x) \pi^{j}(x) x$ with $|x|=1$. Say $x=a \in \Sigma_{3}$. Due to the form of $\mathbf{u}$ we get that between the two occurrences of the letter $x=a$ we must find the two other letters of $\Sigma_{3}$ (that is, both letters from $\Sigma_{3} \backslash\{a\}=\{b, c\}$ ). Indeed $\mathbf{u}$ does not contain a block of 4 consecutive identical letters, so the two occurrences of the letter $x=a$ belong to separate maximal blocks made of letters $x=a$ of the word $\mathbf{u}$, and between two such blocks the other two letters of $\Sigma_{3}$ must occur. But this would mean that $\mathbf{u}$ contains the factor $a b c a$, so $\mathbf{h}$ should contain the factor 11, a contradiction.

The following lemma is immediate, as $\mathbf{v}$ avoids squares.

Lemma 1. The word $\mathbf{v}$ has no factor of the form $x x \pi^{j}(x) \pi^{k}(x), x \pi^{i}(x) \pi^{i}(x) x$, $x \pi^{i}(x) \pi^{j}(x) \pi^{j}(x)$ where $\pi$ is an anti-/morphic permutation and $i, j, k$ are nonnegative integers.

In 8 the following was shown. 
Lemma 2. There exists an infinite word $\mathbf{v}_{m}$ (respectively, $\mathbf{v}_{a}$ ) over $\Sigma_{3}$ that has no factor of the form $x \pi(x) x$, when $\pi$ is replaced by a morphic (respectively, antimorphic) permutation.

The final result we need is from 7 .

Lemma 3. For each pattern $\mathcal{P}=x \pi^{i}(x) \pi^{j}(x) \pi^{k}(x)$, where $i, j, k$ are nonnegative integers, there exists an infinite word $\mathbf{u}_{\mathcal{P}} \in \Sigma_{2}^{\omega}$ (respectively, $\mathbf{u}_{\mathcal{P}}^{\prime} \in$ $\left.\Sigma_{2}^{\omega}\right)$ that does not contain an instance of $\mathcal{P}$ when $\pi$ is replaced by a morphic ${ }_{215}$ (respectively, antimorphic) involution of $\Sigma_{2}$.

We can now show the two main results of this section.

Lemma 4. For each pattern $\mathcal{P}=x \pi^{i}(x) \pi^{j}(x) \pi^{k}(x)$, where $i, j, k$ are nonnegative integers, there exists an infinite ternary word $\mathbf{w}_{\mathcal{P}}$ that does not contain any instance of this pattern with $\pi$ a morphic permutation of $\Sigma_{3}$.

Proof. Clearly, each morphic permutation $\pi$ of $\Sigma_{3}$ is either a cycle or an involution. In all cases, $\pi^{6}$ is the identity morphism on $\Sigma_{3}^{*}$. Consequently, we can replace the exponents $i, j, k$ by their values modulo 6 .

By Corollary 1 and Lemmas 1 and 2 , all the patterns $x \pi^{i}(x) \pi^{j}(x) \pi^{k}(x)$ with one of $i, j, k$ equal to 0 and $\pi$ replaced by a morphic permutation are avoidable, 225 either by $\mathbf{v}$ (when $i=0$ ), either by $\mathbf{v}_{m}$ (when $j=0$ ), or by $\mathbf{u}($ when $k=0$ ). Similarly, the patterns $x \pi^{i}(x) \pi^{j}(x) \pi^{k}(x)$ with $i=k$ are avoided by $\mathbf{v}_{m}$, since this word does not contain instances of any pattern $\pi^{i}(x) \pi^{j}(x) \pi^{i}(x)$, while those with $i=j$ or $j=k$ are avoided by $\mathbf{v}$.

Consequently, we only have to consider the case when $0, i, j, k$ are pairwise distinct, and each is at most 5 in the following.

We look at the reminders of $i, j$ and $k$ modulo 3 .

If $\{1,2\} \subseteq\{i(\bmod 3), j(\bmod 3), k(\bmod 3)\}$, we get that when replacing $\pi$ with a cycle of $\Sigma_{3}$ (e.g., $\pi(0)=1, \pi(1)=2, \pi(2)=0$ ), the instance of $\mathcal{P}=x \pi^{i}(x) \pi^{j}(x) \pi^{k}(x)$ will contain all the three letters 0,1 , and 2 . It follows 235 that $\mathbf{u}_{\mathcal{P}}$ (from Lemma 3 avoids $p$. Indeed, when $\pi$ is replaced by an involution of $\Sigma_{2}$ the result follows from the definition of $\mathbf{u}_{\mathcal{P}}$, while when $\pi$ is replaced 
by any other permutation of $\Sigma_{3}$, its instances will contain the letter 2 , so $\mathbf{u}_{\mathcal{P}}$ canonically avoids all of them.

So, the only case left to consider is when $\{i(\bmod 3), j(\bmod 3), k(\bmod 3)\}$ 240 is either $\{0,1\}$ or $\{0,2\}$. If $i, j, k$ are all equal modulo 3 it follows that at least two of them are actually equal, a contradiction to our earlier assumption that each two of the exponents are different. So, one of $i, j$, and $k$ should be 3 .

It is not hard to see that $x \pi^{i}(x) \pi^{3}(x) \pi^{k}(x)$ is avoided by $\mathbf{v}$. Indeed, an instance of this pattern will always contain a square. In the case when $\pi$ is a cycle of $\Sigma_{3}$ we can only obtain words which have the form $x f(x) x f(x)$ for some morphic permutation $f$ of $\Sigma_{3}$, while for $\pi$ an involution the words we obtain definitely contain an instance of either $x x$ or $\pi(x) \pi(x)$. So, in all cases, the instances of $x \pi^{i}(x) \pi^{3}(x) \pi^{k}(x)$ contain squares. By a similar argument, every pattern $x \pi^{i}(x) \pi^{j}(x) \pi^{3}(x)$ or $x \pi^{3}(x) \pi^{j}(x) \pi^{k}(x)$ is avoided by $\mathbf{v}$, as each instance of such a pattern contains a square.

Lemma 5. For each pattern $\mathcal{P}=x \pi^{i}(x) \pi^{j}(x) \pi^{k}(x)$, where $i, j, k$ are nonnegative integers, there exists an infinite ternary word $\mathbf{w}_{\mathcal{P}}$ that does not contain any instance of this pattern with $\pi$ an antimorphic permutation of $\Sigma_{3}$.

Proof. Just like in the previous proof, for each antimorphic permutation $\pi$ of $255 \Sigma_{3}$, we have that $\pi^{6}$ is the identity morphism on $\Sigma_{3}^{*}$. Consequently, we can replace the exponents $i, j, k$ by their values modulo 6 .

Using Lemma 2, we get that the patterns $x \pi^{i}(x) \pi^{j}(x) \pi^{k}(x)$ with one of $i, j, k$ equal to 0 and $\pi$ replaced by a antimorphic permutation are avoidable, either by $\mathbf{v}$ (when $i=0$ ), either by $\mathbf{v}_{a}$ (when $j=0$ ), or by $\mathbf{u}$ (when $k=0$ ). The patterns $x \pi^{i}(x) \pi^{j}(x) \pi^{k}(x)$ with $i=k$ are avoided by $\mathbf{v}_{a}$, while those with $i=j$ or $j=k$ contain squares, so are avoided by $\mathbf{v}$.

So, just like before, we only have to consider in the following the case when each two of $0, i, j, k$ are distinct and each is at most 5 . And, again, if we have that $\{1,2\} \subseteq\{i(\bmod 3), j(\bmod 3), k(\bmod 3)\}$, we get that when replacing $\pi$ with a cycle of $\Sigma_{3}$ the instance of $\mathcal{P}=x \pi^{i}(x) \pi^{j}(x) \pi^{k}(x)$ will contain all the three letters 0,1 , and 2 . So, by Lemma 3 , it follows that $\mathbf{u}^{\prime} \mathcal{P}$ avoids $\mathcal{P}$. 
Hence, the only case left to consider is when $\{i(\bmod 3), j(\bmod 3), k(\bmod 3)\}$ is either $\{0,1\}$ or $\{0,2\}$. The simple case is again when $i, j, k$ are all equal modulo 3, as it follows that at least two of them are actually equal, which is a contradiction to our assumption that each two of the exponents are different. So, one of $i, j$, and $k$ should be 3 . A more detailed analysis is needed now.

Let us first look at patterns $x \pi^{3}(x) \pi^{j}(x) \pi^{k}(x)$. Obviously, $j$ and $k$ are not of the same parity; actually, the pair $(j, k)$ is one of the pairs $(1,4),(4,1),(2,5),(5,2)$. Generally, when substituting $\pi$ with a cycle of $\Sigma_{3}$, the pattern $x \pi^{3}(x) \pi^{j}(x) \pi^{k}(x)$ equals $x x^{R} \pi^{j}(x) \pi^{k}(x)$. But the instances of $x x^{R} \pi^{j}(x) \pi^{k}(x)$ always contain a square: the last letter of $x$ equals the first letter of $x^{R}$. When substituting $\pi$ with an involution of $\Sigma_{3}$, the pattern $x \pi^{3}(x) \pi^{j}(x) \pi^{k}(x)$ either equals $x \pi(x) x \pi(x)$ if $j$ is even and $k$ is odd, or $x \pi(x) \pi(x) x$ if $j$ is odd and $k$ is even. Also in these cases the instances of the pattern contain squares. So, every instance of the pattern $x \pi^{3}(x) \pi^{j}(x) \pi^{k}(x)$ contains a square. This means that $\mathbf{v}$ avoids $x \pi^{3}(x) \pi^{j}(x) \pi^{k}(x)$.

Next we consider the patterns $x \pi^{i}(x) \pi^{j}(x) \pi^{3}(x)$. Like before, $i$ and $j$ do not have the same parity as $(i, j)$ must be one of the pairs $(1,4),(4,1),(2,5),(5,2)$. Let us assume that $i$ is even and $j$ is odd. If $\pi$ is a cycle, we have that a factor 285 of the form $x \pi^{i}(x) \pi^{j}(x) \pi^{3}(x)$ has the form $x f(x) f\left(x^{R}\right) x^{R}$ for some morphic permutation $f$, which contains the square formed by the last letter of $f(x)$ and the first letter of $f\left(x^{R}\right)$. If $\pi$ is an involution then each factor of the form $x \pi^{i}(x) \pi^{j}(x) \pi^{3}(x)$ starts with $x x$. Therefore, $\mathbf{v}$ avoids $x \pi^{i}(x) \pi^{j}(x) \pi^{3}(x)$ with $i$ even and $j$ odd. Further, we assume that $i$ is odd and $j$ is even. If $\pi$ is a cycle, we have that a factor of the form $x \pi^{i}(x) \pi^{j}(x) \pi^{3}(x)$ has the form $x f\left(x^{R}\right) f(x) x^{R}$ for some morphic permutation $f$, which contains the square formed by the last letter of $f\left(x^{R}\right)$ and the first letter of $f(x)$. If $\pi$ is an involution then each factor of the form $x \pi^{i}(x) \pi^{j}(x) \pi^{3}(x)$ is, in fact, $x \pi(x) x \pi(x)$. So, $\mathbf{v}$ avoids $x \pi^{i}(x) \pi^{j}(x) \pi^{3}(x)$ also for $i$ odd and $j$ even.

Finally, we consider the patterns $x \pi^{i}(x) \pi^{3}(x) \pi^{k}(x)$. Let us assume first that $i$ is odd; consequently, $k$ is even (the pair $(i, k)$ is either $(1,4)$ or $(2,5)$ ). By Theorem 1, the word $\mathbf{u}$ contains no instances of $x \pi^{i}(x) \pi^{3}(x) \pi^{k}(x)$ with $|x| \geq 2$. 
We show that $\mathbf{u}$ does not contain instances of this pattern with $|x|=1$. Assume that $x=a \in \Sigma_{3}$. If $\pi$ is a cycle then the factors $x \pi^{i}(x) \pi^{3}(x) \pi^{k}(x)$ are, in fact,

$a b a b$ with $b \in \Sigma_{3}$ such that $\pi^{i}(x)=b$. If $\pi$ is an involution then the factors $x \pi^{i}(x) \pi^{3}(x) \pi^{k}(x)$ are abba with $b \in \Sigma^{3}$ such that $\pi(x)=b$. By the structure of $\mathbf{u}$ (which has the form $\left(0^{+} 1^{+} 2^{+}\right)^{\omega}$ ), we get that it cannot contain such factors. So $\mathbf{u}$ avoids such patterns.

We now consider the case when $i$ is even and $k$ is odd. Let us write the ThueMorse word as $\mathbf{t}=\prod_{i=0}^{\infty} t_{i}$ with $t_{i} \in\{0,1\}$. Consider the word $\mathbf{t}^{\prime} \in\{0,1\}^{\omega}$ (also used in [7]) given by $\mathbf{t}^{\prime}=\prod_{i=0}^{\infty} 01^{t_{i}+2}$.

We show now that $\mathbf{t}^{\prime}$ avoids $x \pi^{i}(x) \pi^{3}(x) \pi^{k}(x)$ with $i$ even and $k$ odd. If $\pi$ is a cycle then $x \pi^{i}(x) \pi^{3}(x) \pi^{k}(x)$ equals $x f(x) x^{R} f\left(x^{R}\right)$ for some morphic permutation $f$ (which is also a cycle). If $x$ starts with 0 , then $f(x)$ starts with $1, x^{R}$ ends with 0 , and $f\left(x^{R}\right)$ ends with 1 . But 0101 is not a factor $\mathbf{t}^{\prime}$ (there are always at least 2 symbols 1 in a block). Thus, if $\mathbf{t}^{\prime}$ contains an instance of $x f(x) x^{R} f\left(x^{R}\right)$ with $x$ starting with 0 , then $|x| \geq 2$. Now, 0 is always followed by an 1 , so $x$ should start with 01 . This means that $f(x)$ starts with $10, x^{R}$ ends with 10, and $f\left(x^{R}\right)$ ends with 01. Clearly, 01100110 is not a factor of $\mathbf{t}^{\prime}$ (as this infinite word does not contain consecutive 0 letters), so if $\mathbf{t}^{\prime}$ contains an instance of our pattern, then $|x| \geq 3$. Now, as $f\left(x^{R}\right)$ ends with 01 and there are no two consecutive 0 's in $\mathbf{t}^{\prime}$ we get that $f\left(x^{R}\right)$ should end with 101 . This means that $x$ should start with 010, a contradiction, as 1 letters always occur in blocks of length at least 2 . In conclusion $\mathbf{t}^{\prime}$ contains no instance of $x \pi^{i}(x) \pi^{3}(x) \pi^{k}(x)$ with $x$ starting with 0 and $\pi$ an antimorphic cycle; analogously, one can show that $\mathbf{t}^{\prime}$ contains no instance of $x \pi^{i}(x) \pi^{3}(x) \pi^{k}(x)$ with $x$ starting with 1 and $\pi$ an antimorphic cycle. Moreover, by a very similar analysis one can show that $\mathbf{t}^{\prime}$ does not contain any instance of $x \pi^{i}(x) \pi^{3}(x) \pi^{k}(x)$ with $\pi$ an antimorphic involution. We have, thus, shown that $\mathbf{t}^{\prime}$ avoids the pattern $x \pi^{i}(x) \pi^{3}(x) \pi^{k}(x)$ with $i$ even and $k$ odd.

This concludes the proof of this lemma.

By Lemmas 4 and 5 we obtain the following theorem. 
Theorem 3. All patterns $x \pi^{i}(x) \pi^{j}(x) \pi^{k}(x)$, where $i, j, k$ are non-negative integers, and $\pi$ is substituted by an anti-/morphic permutation, are avoidable over $\Sigma_{3}$.

We conclude this section with the following result, which follows from the previous theorem by the arguments already presented in the end of Section 2 .

Theorem 4. All patterns $\pi^{i_{1}}(x) \pi^{i_{2}}(x) \ldots \pi^{i_{r}}(x)$ with $r \geq 4$, the $i_{j}$ non-negative integers, and $\pi$ an anti-/morphic permutation, are avoidable over $\Sigma_{3}$.

\subsection{Four and five letter alphabets: the morphic case}

In this section we extend the result of the previous sections, and we show that all patterns $x \pi^{i}(x) \pi^{j}(x) \pi^{k}(x)$, with $i, j, k \geq 0$, are avoidable in $\Sigma_{4}$ and at least one pattern of this form becomes unavoidable in $\Sigma_{5}$, when $\pi$ is substituted by a morphic permutation.

Let us consider a pattern $x \pi^{i}(x) \pi^{j}(x) \pi^{k}(x)$, with $i, j, k \geq 0$. For simplicity, the factors $x, \pi^{i}(x), \pi^{j}$, or $\pi^{k}(x)$ of the pattern are called $x$-items in the following. Our analysis is based on the relation between the possible images of the four $x$-items occurring in a pattern, following the ideas of [8]. For instance, we want to check whether in a possible image of our pattern, all four $x$-items can be mapped to a different word, or whether the second $x$-item and the last one can be mapped to the same word, and so on.

To achieve this, we define in Table 4.2 the numbers $k_{i}$, with $1 \leq i \leq 14$. Intuitively, they allow us to define, for a pattern $x \pi^{i}(x) \pi^{j}(x) \pi^{k}(x)$, which are the alphabets $\Sigma_{m}$ in which we can model certain (non-)equality relationships between the images of the $x$-items. For example, in alphabets $\Sigma_{m}$ with $m \geq k_{1}$ we can assign values to $x$ and $\pi$ such that the images of every two of $\pi^{i}(x), \pi^{j}(x)$, and $\pi^{k}(x)$ are different (and this property does not hold in alphabets with less than $k_{1}$ letters), while for $\Sigma_{m}$ with $m \geq k_{2}$ we can assign values to $x$ and $\pi$ such that the images of $x$ and $\pi^{i}(x)$ are equal to some word, while the images of $355 \pi^{j}(x)$ and $\pi^{k}(x)$ are assigned to two other words (also different between them; again, this property does not hold in smaller alphabets). To simplify, we use 


\begin{tabular}{|l|l|}
\hline$k_{1}=\inf \{t: t \nmid i, t \nmid j, t \nmid k, t \nmid|i-j|, t \nmid|i-k|, t \nmid|j-k|\}$ & 0123 \\
\hline$k_{2}=\inf \{t: t|i, t \nmid j, t \nmid k, t \nmid| j-k \mid\}$ & 0012 \\
\hline$k_{3}=\inf \{t: t \nmid i, t|j, t \nmid k,, t \nmid| i-k \mid\}$ & 0102 \\
\hline$k_{4}=\inf \{t: t \nmid i, t \nmid j, t|| i-k \mid\}$ & 0121 \\
\hline$k_{5}=\inf \{t: t \nmid i, t \nmid j, t \nmid|i-j|, t \nmid|i-k|, t|| j-k \mid\}$ & 0122 \\
\hline$k_{6}=\inf \{t: t|i, t| j, t \nmid k\}$ & 0001 \\
\hline$k_{7}=\inf \{t: t|i, t \nmid j, t| k\}$ & 0010 \\
\hline$k_{8}=\inf \{t: t \nmid i, t|j, t| k\}$ & 0100 \\
\hline$k_{9}=\inf \{t: t \nmid i, t|| i-j|, t||i-k|\}$ & 0111 \\
\hline$k_{10}=\inf \{t: t|i, t \nmid j, t||j-k|\}$ & 0011 \\
\hline$k_{11}=\inf \{t: t \nmid i, t|j, t||i-k|\}$ & 0101 \\
\hline$k_{12}=\inf \{t: t \nmid i, t|k, t||i-j|\}$ & 0110 \\
\hline$k_{13}=\inf \{t: t \nmid i, t \nmid k, t|| i-j \mid\}$ & 0112 \\
\hline$k_{14}=\inf \{t: t \nmid i, t \nmid j, t|| i-j \mid\}$ & 0120 \\
\hline & \\
\hline
\end{tabular}

Table 1: Definition of the values $k_{i}$, with $1 \leq i \leq 14$. We set $K=\left\{k_{1}, k_{2}, \ldots, k_{14}\right\}$.

a simple digit-representation for any of these cases, defined in the last column of Table 4.2. In this representation, we assign different digits to the $x$-items that can be mapped to different words. For example, we use the representation 0123 for the case defined through $k_{1}$ and 0012 for the case defined by $k_{2}$. In general, when considering a $k_{i}$, we assign a 4 -digit representation to the pattern $x \pi^{i}(x) \pi^{j}(x) \pi^{k}(x)$ in the following manner: we start with 0 , and then put a 0 on all of the remaining three positions corresponding to an $x$-item $\pi^{t}(x)$ to such that $k_{i} \mid t$. We then put a 1 on the the leftmost empty position. If the $x$-item on the respective position is $\pi^{r}(x)$, we put 1 on all empty positions $s$ such that $k_{i} \mid(r-s)$, and so on.

Recall that $\inf \emptyset=\infty$, so some of the $k_{i}$ s may be infinite. However, note that the set $\{t: t|i, t| j, t|k, t||i-j|, t|| i-k|, t||j-k|\}$ defining $k_{1}$ is always non-empty, and also that $k_{1}>3$. Indeed, at least two of $i, j, k$ have 

two have the same reminder modulo 3 , so $k_{1}$ should also not divide 3 . Let $K=\left\{k_{1}, k_{2}, \ldots, k_{14}\right\}$.

For a pattern $x \pi^{i}(x) \pi^{j}(x) \pi^{k}(x)$, we say that some $k_{i}$ and the digit-string encoding it model an instance $u f^{i}(u) f^{j}(u) f^{k}(u)$ of the pattern if each two of the factors $u, f^{i}(u), f^{j}(u), f^{k}(u)$ are equal if and only if the digits corresponding to the respective factors in the digit representation of that $k_{i}$ are equal.

Lemma 6. The pattern $x \pi^{i}(x) \pi^{j}(x) \pi^{k}(x)$, with $i \neq j \neq k \neq i$ is unavoidable in $\Sigma_{m}$, for $m \geq \max \left\{k_{1}, k_{3}, k_{6}, k_{12}, k_{13}\right\}$.

ProOF. Let $p=x \pi^{i}(x) \pi^{j}(x) \pi^{k}(x)$.

Because $m \geq k_{1}$, we have that for every word $u \in \Sigma_{m}^{+}$there exists a morphic permutation $f$ such that every two words of $u, f^{i}(u), f^{j}(u), f^{k}(u)$ are different. Indeed, we take $f$ to be a permutation such that the orbit of $u[1]$ is a cycle of length $k_{1}$, which means that the the first letters of $u, f^{i}(u), f^{j}(u)$ and $f^{k}(u)$ are pairwise different. Similarly, the fact that $m \geq k_{3}$ (when $k_{3} \neq \infty$ ) means that $f^{j}(u) \neq f^{k}(u) \neq f^{i}(u)$. In this case, we take $f$ to be a permutation such that $\operatorname{ord}_{f}(u[1])=k_{3}$. We can derive similar observations for $k_{6}, k_{12}$, and $k_{13}$, as well as for all the $k_{i}$ values we defined.

One can check with the aid of a computer, by a straightforward backtracking algorithm, that if $m \geq \max \left\{k_{1}, k_{3}, k_{6}, k_{12}, k_{13}\right\}$ then the longest word over $\Sigma_{m}$ that does not contain an instance of this pattern has length 14 . Our computer program (available at https://www.dropbox.com/s/5q5yavloti9nm3h/ lemma6.rb) tries construct a word as long as possible by always adding a letter to the current word it constructed by backtracking; this letter is chosen in all possible ways from the letters contained in the word already, or it may also be a new letter. An example of one of the longest words our programe constructed, over an alphabet of size greater or equal to $m$, which is at its turn greater or equal to 3 as $k_{1} \geq 3$, is 00120120120111 (adding new letters to this word does not lead to a longer one). This concludes our proof. 
In the following Lemmas we show a series of avoidability and unavoidability results. Our first result uses the morphism $\alpha: \Sigma_{3}^{*} \rightarrow \Sigma_{4}^{*}$ that is defined by

$$
0 \rightarrow 01220112, \quad 1 \rightarrow 0, \quad 2 \rightarrow 03110223
$$

Lemma 7. Consider the infinite word:

$$
\boldsymbol{h}_{\alpha}=\alpha(\boldsymbol{h})=0122011200311022301220112031102230012201120031102230 \ldots
$$

If $\boldsymbol{h}_{\alpha}$ contains an instance of the pattern $x \pi^{i}(x) \pi^{j}(x) \pi^{k}(x)$ then this instance is not modelled by any element of the set

$$
\left\{k_{3}, k_{4}, k_{6}, k_{7}, k_{8}, k_{9}, k_{10}, k_{11}, k_{12}, k_{14}\right\} \text {. }
$$

Proof. The maximum length of a factor of $\mathbf{h}_{\alpha}$ that does not contain a full image of a letter 2 from the Hall word under $\alpha$ is 24 . Using a computer program (available at https://www.dropbox.com/s/faeyam3lb5kky59/lemma7.rb) we checked that, there is no factor of the form $u f(u) g(u) h(u)$ with $|u|<25$ which can be modelled by any of the $k_{i}$ s mentioned above (with $f, g$, and $h$ morphic permutations). By this we mean that there is no factor $u f(u) g(u) h(u)$ of $\mathbf{h}_{\alpha}$, with $|u|<25$, such that two of the factors $u, f(u), g(u), h(u)$ are equal if and only if the digits on their respective positions (i.e., 1, 2, 3, and, respectively, 4) in $k_{i}$ are equal. Further, if $u$ is a word of length $\geq 25$, and $\mathbf{h}_{\alpha}$ contains an instance $u f^{i}(u) f^{j}(u) f^{k}(u)$ of the pattern $x \pi^{i}(x) \pi^{j}(x) \pi^{k}(x)$, then $u$ contains the factor 3110223. Based on the repetitions of 1,2 and 3, the factor 3110223 of $u$ should be aligned with some factors of the form abbcdda from $f^{i}(u), f^{j}(u)$ and $f^{k}(u)$, respectively. The only possible such alignment is to align 3110223 from $u$ with other occurrences of 3110223 . This means that $f^{i}, f^{j}, f^{k}$ are all the identity, so $\mathbf{h}_{\alpha}$ contains a 4-power $u^{4}$, with $|u| \geq 25$. Looking at the occurrence of $u^{4}$ in $\mathbf{h}_{\alpha}$ we get that the $i^{\text {th }}$ occurrence of $u$ in this repetition can be written as $u_{i}^{\prime} h\left(x_{1, i}\right) h\left(x_{2, i}\right) \ldots h\left(x_{t, i}\right) u_{i}^{\prime \prime}$ where $1 \leq i \leq 4, x_{1, i}, \ldots, x_{k, i} \in\{0,1,2\}$ and, for $1 \leq j \leq 3$ we have $u_{j}^{\prime \prime} u_{j+1}^{\prime}=h\left(x_{j}\right)$ for some letter $x_{i} \in\{0,1,2\}$. As the image of each letter under $h$ starts with 0 , and none starts with 02 nor with 011, we get that $u_{1}^{\prime}=u_{2}^{\prime}=u_{3}^{\prime}=u_{4}^{\prime}$. Thus, $u_{1}^{\prime}=u_{2}^{\prime \prime}=u_{3}^{\prime \prime}$ (so, $x_{1}=x_{2}$ as well), ${ }_{425}$ and $x_{j, 1}=x_{j, 2}$ for $1 \leq j \leq k$. Accordingly, $\mathbf{h}$ should contain a square, which is a contradiction. 
Lemma 8. Let $K^{\prime}=\left\{k_{i_{1}}, k_{i_{2}}, k_{i_{3}}\right\} \subset K$ be any subset of size 3 of $K$. There exists an infinite word $w$ such that $w$ does not contain 4-powers and if $w$ contains an instance of the pattern $x \pi^{i}(x) \pi^{j}(x) \pi^{k}(x)$ then it can not be modelled by any tuples of the set of patterns $K^{\prime}$.

Proof. We consider all possible combinations of size three of $k_{i} \mathrm{~s}$, and we check the avoidability of each combination.

To begin with, assume that $k_{i_{1}}, k_{i_{2}}, k_{i_{3}}$ are all $k_{i} \mathrm{~s}$ whose representations contain at least three different digits. Then if the word $\mathbf{t}$ contains an instance of ${ }_{435}$ the pattern $x \pi^{i}(x) \pi^{j}(x) \pi^{k}(x)$, it can not be modelled by any $k_{i} \in K^{\prime}$, as such a $k_{i}$ can model only instances of the pattern over an alphabet of size greater or equal to 3 .

Assume now $k_{i_{1}}, k_{i_{2}}, k_{i_{3}}$ are all $k_{i} \mathrm{~s}$ whose representations contain at most two different digits (e.g., $k_{6}, k_{7}$, etc.). Then if the word $\mathbf{h}$ contains an instance of the pattern $x \pi^{i}(x) \pi^{j}(x) \pi^{k}(x)$, it can not be modelled by any $k_{i} \in K^{\prime}$, since $\mathbf{h}$ does not contain any square, but these $k_{i}$ s can only model instances of the pattern that contain at least one square.

If two of $k_{i_{1}}, k_{i_{2}}, k_{i_{3}}$ are among the $k_{i}$ s whose representation has at least three different digits, and the other one of them is a $k_{i}$ whose representation has at most two different digits, then, similar to the previous section, we can show that $\mathbf{u}_{\mathcal{P}}$ (from Lemma 3) does not contain any instance of pattern that can be modelled by the respective $k_{i}$. Indeed, this word does not contain instances of the pattern modelled by the any $k \in K$ that can be represented with only two digits (as such an instance could also be modelled with the restriction that $\pi$ is replaced by an involution), and the remaining two $k_{i} \mathrm{~s}$ can, once more, model only instances of the pattern over an alphabet greater or equal to 3 .

Assume two of $k_{i_{1}}, k_{i_{2}}, k_{i_{3}}$ are any of the $k_{i}$ s whose representation has at most two different digits, and one of them is any $k_{i}$ except $k_{3}, k_{4}, k_{14}$, which is represented using at least three different digits. Then $\mathbf{h}$ does not contain instances of patterns that can be modelled by any $k_{i} \in K^{\prime}$, because all such $k_{i}$ s model only instances of the pattern that contain squares or have 4 different 
letters (e.g., the instances modeled by $k_{1}$ ).

Assume two of $k_{i_{1}}, k_{i_{2}}, k_{i_{3}}$ are any of $k_{i} \mathrm{~s}$ whose representation has at most two different digits, and one of them is $k_{3}$ or $k_{4}$ or $k_{14}$, the word defined in Lemma 7 can avoid them.

Lemma 9. For each pattern $\mathcal{P}=x \pi^{i}(x) \pi^{j}(x) \pi^{k}(x)$, where $i, j, k$ are nonnegative integers, there exists an infinite word $\boldsymbol{w}_{p}$ that does not contain any instance of this pattern with $\pi$ a morphic permutation of $\Sigma_{4}$.

Proof. In this proof, we do a case analysis depending on the possible permutations of $\Sigma_{4}$ that $\pi$ can be assigned to.

If $\pi$ is assigned to the identical permutation $1_{\Sigma_{4}}$, then the image obtained is a 4-power. If $\pi$ is assigned to a 4-cycle $f$, then any instance $u f^{i}(u) f^{j}(u) f^{k}(u)$ is, actually, equal to the word $u f^{i \bmod 4}(u) f^{j \bmod 4}(u) f^{k \bmod 4}(u)$. In this case, if we have two different exponents then also the factors corresponding to them are different. Accordingly, these instances (which are not 4-powers) are modelled by exactly one $k_{i}$, called $k_{i_{1}}$ in the following. If $\pi$ is assigned a permutation $f$ that permutes in a cycle three elements of $\Sigma_{4}$ and fixes the remaining one, then any instance $u f^{i}(u) f^{j}(u) f^{k}(u)$ is either a 4-power, if $u=a^{k}$ and $a$ is the fixed point of $f$, or $u f^{i} \bmod 3(u) f^{j} \bmod 3(u) f^{k} \bmod 3(u)$, otherwise. Again these instances are modelled by exactly one $k_{i}$, called $k_{i_{2}}$ in the following, and a 4-power. Assume $\pi$ is mapped to a permutation $f$ that is the composition of two disjoint cycles of length 2 , or $f$ consists of a cycle of length 2 and two fixed points (in other words, $f$ is an involution). Then any instance $u f^{i}(u) f^{j}(u) f^{k}(u)$ is either the identity, if $u \in\{a, b\}^{*}$ and $a, b$ are fixed point of $f$, or it is equal to $u f^{i \bmod 2}(u) f^{j \bmod 2}(u) f^{k \bmod 2}(u)$, otherwise. Yet again, these instances are modelled by exactly one $k_{i}$, called $k_{i_{3}}$, and a 4 -power.

Our statement follows now from Lemma 8, as there exists a word that does not contain any instance of the pattern that is a 4-power or modelled by $\left\{k_{i_{1}}, k_{i_{2}}, k_{i_{3}}\right\}$.

As an example, for $i=3, j=16, k=2$, if $\pi$ is mapped to a 4-cycle, we can obtain instances which are 4-powers or are modelled by 0102. If $\pi$ is mapped to 
a 3-cycle and a fixed point, the instances we obtain are 4-powers or are modelled by 0012 , and if $\pi$ is mapped to an involution, we can obtain instances which are 4-powers or are modelled by 0100 . All such instances can be avoided by a single infinite word, according to Lemma 8

According to the previous lemmas, we can now prove the following theorems.

Theorem 5. All patterns $x \pi^{i}(x) \pi^{j}(x) \pi^{k}(x)$, where $i, j, k$ are non-negative integers, and $\pi$ is substituted by a morphic permutation, are avoidable over $\Sigma_{4}$.

Theorem 6. All patterns $\pi^{i_{1}}(x) \pi^{i_{2}}(x) \ldots \pi^{i_{r}}(x)$ with $r \geq 4$, the $i_{j}$ non-negative integers, and $\pi$ a morphic permutation, are avoidable over $\Sigma_{4}$.

The above results are optimal, in the sense that they cannot be extended for $\Sigma_{5}$. This is shown by the next lemma.

Lemma 10. There exists a pattern $x \pi^{i}(x) \pi^{j}(x) \pi^{k}(x)$, with $i \neq j \neq k \neq i$ which is unavoidable in $\Sigma_{m}$, for $m \geq 5$.

Proof. To show that there exists a pattern $x \pi^{i}(x) \pi^{j}(x) \pi^{k}(x)$ which is unavoidable in $\Sigma_{m}$ it is enough to find $i, j$ and $k$ such that the instances of the pattern $x \pi^{i}(x) \pi^{j}(x) \pi^{k}(x)$ are all modelled by the set $\left\{k_{1}, k_{3}, k_{6}, k_{12}, k_{13}\right\}$, from Lemma 6. To this end, let us consider the pattern $x \pi^{2}(x) \pi^{56}(x) \pi^{33}(x)$. We show that this pattern is unavoidable in $\Sigma_{5}$. Indeed, if $\pi$ is mapped to a 5 -cycle, the instances we obtain are modelled by 0123 . If $\pi$ is mapped to a permutation composed of a 4-cycle and a fixed point, the instances we obtain are modelled by 0102. If $\pi$ is mapped to an involution, the instances we obtain are modelled by 0001. If $\pi$ is mapped to the composition of a 3 -cycle and two fixed points, then the words we obtain are modelled by 0110. Finally, if $\pi$ is mapped to a permutation which is the composition of a 3-cycle and a 2-cycle (so, which has order 6), then the instances of the pattern are modelled by 0112. Putting all these together, we obtain that the instances of the pattern are all modelled by the set $\left\{k_{1}, k_{3}, k_{6}, k_{12}, k_{13}\right\}$. By Lemma 6, we get that the pattern $x \pi^{2}(x) \pi^{56}(x) \pi^{33}(x)$ is unavoidable. 
Thus, we have shown the following theorem.

Theorem 7. The largest $m$ such that all patterns $x \pi^{i}(x) \pi^{j}(x) \pi^{k}(x)$, with $i, j, k>$ 0 and $\pi$ morphic permutation, are avoidable in $\Sigma_{m}$ is $m=4$.

\section{Eventually unavoidable patterns}

In the final section of this paper, we show that for all $n \geq 1$ there exists 520 a pattern under permutations of length $n$ and an alphabet $\Sigma$ such that the respective pattern is unavoidable over $\Sigma$.

Let $n$ be a positive integer, and let $i_{j}$ be non-negative integers, $0 \leq j \leq n-1$. Consider the unary pattern of length $n$ given by

$$
\mathcal{P}=\pi^{i_{1}}(x) \pi^{i_{2}}(x) \cdots \pi^{i_{n-1}}(x) \pi^{i_{n}}(x)
$$

We say that $\mathcal{P}$ is eventually unavoidable if there exists an integer $N$ such that, whenever $\Sigma$ is an alphabet with $|\Sigma| \geq N$, and $w \in \Sigma^{n}$, there is a permutation $\pi$ of $\Sigma$ and a letter $a \in \Sigma$, such that

$$
w=\pi^{i_{1}}(a) \pi^{i_{2}}(a) \cdots \pi^{i_{n-1}}(a) \pi^{i_{n}}(a) .
$$

Theorem 8. Let $n$ be a non-negative integer. There is an eventually unavoidable pattern of length $n$.

Proof. Consider all partitions of $\{1,2, \ldots, n\}$ into non-empty subsets; there are $B_{n}$ of these, where $B_{n}$ is the $n$th Bell number. Let the $r$ th such partition be

$$
P_{r}=\left\langle A_{1, r}, A_{2, r}, \ldots, A_{j_{r}, r}\right\rangle,
$$

${ }_{525}$ where $\{1,2, \ldots, n\}=A_{1, r} \dot{\cup} A_{2, r} \dot{\cup} \ldots \dot{\cup} A_{j_{r}, r}$. We may assume that the sets $A_{\ell, r}$ of the partition are ordered in increasing order of their least element.

For $1 \leq k \leq n, 1 \leq r \leq B_{n}$, let $q_{k, r}$ be the integer such that $k \in A_{q_{k, r}+1, r}$. In other words, $k$ is in the $\left(q_{k, r}+1\right)$ st piece of the $r$ th partition. Let $p_{m}$ denote the $m$ th prime number. 
For $1 \leq k \leq n$, consider the system of congruences

$$
i_{k} \equiv q_{k, r}\left(\bmod p_{r}\right), 1 \leq r \leq B_{n} .
$$

By the Chinese Remainder Theorem, choose $i_{k}$ satisfying these congruences. (We remark in passing that $i_{0}=0$ is always possible, since 1 is always in the first piece of each partition, by our notational choice.) Let

$$
\mathcal{P}=\pi^{i_{1}}(x) \pi^{i_{2}}(x) \cdots \pi^{i_{n-1}}(x) \pi^{i_{n}}(x)
$$

Let $N=p_{B_{n}}$. Suppose $|\Sigma| \geq N$, and $w \in \Sigma^{n}$.

Suppose $w$ contains exactly $m$ distinct letters; say $w \in T^{n}$, where $|T|=m$. Let $f: T \rightarrow\{1,2, \ldots, m\}$ be given by $f(x)=\ell$ if $x$ first occurs on position $i$ of $w$ and the number of distinct letters occuring in the length $i-1$ prefix of $w$ is exactly $\ell-1$. For instance, if $T=\{a, b, c\}$, and $w=a a c a b a b b$, then $f(a)=1$, $f(b)=3$, and $f(c)=2$. In other words, $f^{-1}$ encodes the order in which the letters of $T$ occur in $w$.

We canonically extend $f$ to a morphism $f: T^{*} \rightarrow\{1, \ldots, m\}^{*}$. In our previous example, $f(w)=11213133$. Note that the first letter of $f(w)$ is always 1.

We will show that there is a permutation $\pi \in S_{N}$, such that

$$
f(w)=\pi^{i_{1}}(1) \pi^{i_{2}}(1) \cdots \pi^{i_{n-1}}(1) \pi^{i_{n}}(1) .
$$

540 The desired result follows, replacing $\pi$ by $f^{-1} \pi f$.

To find the permutation $\pi$, let $P=\left\langle A_{1}, A_{2}, \ldots A_{m}\right\rangle$, where $\ell \in A_{j}$ if and only if the $\ell$ th letter of $f(w)$ is $j$. For some $r, P=P_{r}$. Since $m, p_{r} \leq p_{B_{n}}=N$, $S_{N}$ will contain a $p_{r}$-cycle, $\pi$ such that $\pi=(1,2, \ldots, m, \ldots)$. Here the elements other than the first $m$ can be arbitrary distinct elements of $\{m+1, m+2, \ldots, N\}$.

Now $\pi^{j}(1)=j+1, j=0,1, \ldots, m-1$. Since $\pi$ is a $p_{r}$-cycle, if $i_{k} \equiv$ $q_{k, r}\left(\right.$ modulo $\left.p_{r}\right)$, then $\pi^{i_{k}}(1)=\pi^{q_{k, r}}(1)=q_{k, r}+1$.

The $k^{\text {th }}$ letter of $\pi^{i_{1}}(1) \pi^{i_{2}}(1) \cdots \pi^{i_{n-1}}(1) \pi^{i_{n}}(1)$ is $\pi^{i_{k}}(1)$, which is $q_{k, r}+1$. However, by definition of the $q_{k, r}$, this means that $k$ is in the $\left(q_{k, r}+1\right)$ st piece of $P$. By the definition of $P$, the $k^{t h}$ letter of $f(w)$ is $q_{k, r}+1$. Since $k$ was 
arbitrary, we conclude that $\pi^{i_{1}}(1) \pi^{i_{2}}(1) \cdots \pi^{i_{n-1}}(1) \pi^{i_{n}}(1)=f(w)$, as claimed.

Example 1. Let $n=3$. The partitions of $\{1,2,3\}$ are

$$
\begin{aligned}
& P_{1}=\langle\{1,2,3\}\rangle, P_{2}=\langle\{1\},\{2,3\}\rangle, P_{3}=\langle\{1,2\},\{3\}\rangle, \\
& P_{4}=\langle\{1,3\},\{2\}\rangle, P_{5}=\langle\{1\},\{2\},\{3\}\rangle .
\end{aligned}
$$

This gives

$$
\begin{aligned}
& q_{1,1}=0, q_{2,1}=0, q_{3,1}=0, \quad q_{1,2}=0, q_{2,2}=1, \quad q_{3,2}=1, \\
& q_{1,3}=0, q_{2,3}=0, q_{3,3}=1, \quad q_{1,4}=0, q_{2,4}=1, \quad q_{3,4}=0, \\
& q_{1,5}=0, q_{2,5}=1, q_{3,5}=2 .
\end{aligned}
$$

As mentioned, we can always choose $i_{1}=0$. For $i_{2}$, we get these congruences:

$$
\begin{aligned}
& i_{2} \equiv 0 \quad(\bmod 2), i_{2} \equiv 1(\bmod 3), i_{2} \equiv 0(\bmod 5) \\
& i_{2} \equiv 1 \quad(\bmod 7), i_{2} \equiv 1(\bmod 11)
\end{aligned}
$$

and $i_{2}=2080$ is the smalleast integer solution.

For $i_{3}$, we get these congruences:

$$
\begin{aligned}
& i_{3} \equiv 0 \quad(\bmod 2), i_{3} \equiv 1(\bmod 3), i_{3} \equiv 1(\bmod 5) \\
& i_{3} \equiv 0 \quad(\bmod 7), i_{3} \equiv 2(\bmod 11)
\end{aligned}
$$

and $i_{3}=1036$ is the smalleast integer solution.

We conclude that $x \pi^{2080}(x) \pi^{1036}(x)$ is eventually unavoidable. As soon as $|\Sigma| \geq 11$, any length 3 word encounters this pattern. For example, to see that $w=a b a$ encounters the pattern, we look at $f(w)=121$, and the partition $P=\langle\{1,3\},\{2\}\rangle=P_{4}$. We thus let $\pi$ be a 7 -cycle $\pi=(1,2, \ldots)$. Now $\pi^{2080}(1)=\pi^{2080(\bmod 7)}(1)=\pi(1)=2$, while $\pi^{1036}(1)=\pi^{1036(\bmod 7)}(1)=\pi^{0}(1)=1$, so that $\pi^{0}(1) \pi^{2080}(1) \pi^{1036}(1)=121 \sim a b a$.

By Theorem 8 the following result is immediate. 
$n$ and an integer $N$ such that for all alphabets $\Sigma$ with $|\Sigma| \geq N$, the pattern $\mathcal{P}$ is unavoidable over $\Sigma$.

This last theorem highlights the main open problem of this work. Each pattern under anti-/morphic permutations is avoidable in $\Sigma_{3}$, but some patterns for a given pattern $\mathcal{P}$ which are the alphabets $\Sigma_{k}$ in which it is avoidable? Note that such a result was obtained in [8] for cubic patterns.

\section{References}

[1] J. D. Currie, F. Manea, D. Nowotka, Unary patterns with permutations, in: Developments in Language Theory, Vol. 9168 of Lecture Notes in Computer Science, Springer, 2015, pp. 191-202.

[2] A. Thue, Über unendliche Zeichenreihen, Norske Vid. Skrifter I. Mat.Nat. Kl., Christiania 7 (1906) 1-22.

[3] A. Thue, Über die gegenseitige Lage gleicher Teile gewisser Zeichenreihen, Norske Vid. Skrifter I. Mat.-Nat. Kl., Christiania 1 (1912) 1-67.

[4] M. Lothaire, Combinatorics on Words, Cambridge University Press, 1997.

[5] J. Currie, Pattern avoidance: themes and variations, Theoret. Comput. Sci. 339 (1) (2005) 7-18. doi:10.1016/j.tcs.2005.01.004.

[6] E. Chiniforooshan, L. Kari, Z. Xu, Pseudopower avoidance, Fundam. Inform. 114 (1) (2012) 55-72.

[7] B. Bischoff, J. Currie, D. Nowotka, Unary patterns with involution, Int. J. Found. Comput. Sci. 23 (8) (2012) 1641-1652. doi:10.1142/ S0129054112400679.

[8] F. Manea, M. Müller, D. Nowotka, The avoidability of cubes under permuta585 tions, in: Developments in Language Theory, Vol. 7410 of LNCS, Springer, 2012, pp. 416-427, extended version to appear in J. Comput. Syst. Sci. 
[9] M. Hall, Lectures on Modern Mathematics, Vol. 2, Wiley, New York, 1964, Ch. Generators and relations in groups - The Burnside problem, pp. 42-92. 